INPLASY

PROTOCOL

To cite: Zhang et al. Intervention for reducing anxiety during screening mammography: A protocol for systematic review and metaanalysis. Inplasy protocol 202070131. doi:

10.37766/inplasy2020.7.0131

Received: 31 July 2020

Published: 31 July 2020

Corresponding author:

Zhi-Gang Zhang

2669291698@qq.com

Author Affiliation:

Lanzhou University First

Affiliated Hospital

Support: No.2018-RC-52.

Review Stage at time of this submission: Preliminary searches.

Conflicts of interest:

All authors declare no conflict of interest.

\section{Intervention for reducing anxiety during screening mammography: A protocol for systematic review and meta-analysis}

Zhang, ZG1; Yang, LP2; Shang, Y3; Song, ZW4; Du, L4.

Review question / Objective: How's the effect, patient acceptance, perceived value associated with interventions for reducing anxiety compared to no intervention in women ages 40 and older during screening mammography.

Condition being studied: Among women, breast cancer is a leading cause of death, being the common type of cancer. As we all know, mammography screening can diminish breast cancer mortality by detecting early-stage tumors. The impact of a positive mammography result on women's mental health is an active area of research. Anxiety is, however, often cited as a harm of screening mammography with few options offered to reduce anxiety other than not screening. Diminishing anxiety may improve adherence, and reduce breast cancer mortality and morbidity. This study was conducted to investigate the effects of interventions on level of anxiety among women who are undergoing screening mammography.

INPLASY registration number: This protocol was registered with the International Platform of Registered Systematic Review and Meta-Analysis Protocols (INPLASY) on 31 July 2020 and was last updated on 31 July 2020 (registration number INPLASY202070131).

\section{INTRODUCTION}

Review question / Objective: How's the effect, patient acceptance, perceived value associated with interventions for reducing anxiety compared to no intervention in women ages 40 and older during screening mammography.
Condition being studied: Among women, breast cancer is a leading cause of death, being the common type of cancer. As we all know, mammography screening can diminish breast cancer mortality by detecting early-stage tumors. The impact of a positive mammography result on 
women's mental health is an active area of research. Anxiety is, however, often cited as a harm of screening mammography with few options offered to reduce anxiety other than not screening. Diminishing anxiety may improve adherence, and reduce breast cancer mortality and morbidity. This study was conducted to investigate the effects of interventions on level of anxiety among women who are undergoing screening mammography.

\section{METHODS}

Participant or population: Women aged $\mathbf{4 0}$ and older and undergoing screening mammography.

Intervention: Psychological Interventions and other interventions are included except Pharmacological treatment.

Comparator: No-treatment, regular treatment or nursing.

Study designs to be included: Randomized Controlled Trials (RCTs) will be adopted.

Eligibility criteria: 1 . Women could be of ethnic origin, and the patient have to undergo screening mammography Women aged 40 and older who do NOT have: a history of previous breast cancer or DICS (Ductal carcinoma in situ); a current psychiatric diagnosis; any form of psychiatric treatment now. 2. Published literature. 3. Intervention measures: Interventions using psychological intervention as a main variable. Any comparisons between a combined therapy of psychological intervention and other interventions and a therapy of solely using other interventions are also included. 4. The control group will be no-treatment, regular treatment or Nursing.

Information sources: We will search, with no time restrictions, the following databases for relevant English language literature: PubMed, EMBASE and Cochrane Library. The electronic database search will be supplemented by manual search of the reference lists of included articles.
Main outcome(s): Impact on patient anxiety -Spielberger State Trait Anxiety Inventory (STAI). State anxiety will be measured using the State Anxiety (SA) subscale of the State-Trait Anxiety Inventory (STAI) before and after mammography in both intervention and control groups. Items will be rated on a 0 to 3 Likert-type scale with scores calculated as item sums with a possible range of $0-60$, higher scores indicating greater state anxiety.

Quality assessment / Risk of bias analysis: Two reviewers will independently assess the quality of selected studies according to the Cochrane Collaboration's tool for randomized controlled trials. Items will be evaluated in three categories: Low risk of bias, unclear bias and high risk of bias. The following characteristics will be evaluated: Random sequence generation (selection bias), Allocation concealment (selection bias), Blinding of participants and personnel (performance bias), Incomplete outcome data (attrition bias), Selective reporting (reporting bias), Other biases Results from these questions will be graphed and assesses using Review Manager 5.4.

Strategy of data synthesis: Risk ratio (RR) for both fixed and random effects models (weighting by inverse of variance) will be used. Between-study heterogeneity will be assessed using the $P$ value and 12 statistics. According to the Cochrane handbook, the 12 will be considered nonimportant ([? 30\%), moderate (30\%-60\%) and substantial (?60\%). Results will be assessed using forest plots and presented as RRs for the main outcome and second outcomes. Statistical analysis will be conducted using the statistical package (RevMan v5.4) or Windows.

Subgroup analysis: If there is significant heterogeneity in the included trials, subgroup analysis will be carried out. According to subject characteristics (e.g., level of anxiety, age, gender, and so on), subgroup analysis will be carried out according to the data retrieved. 
Sensibility analysis: If there is still significant heterogeneity in the included trials after subgroup analysis, Sensitivity analysis will be performed to assist exploring the source of heterogeneity. It will be carried out by deleting each study at a time, and other studies will be analyzed to estimate whether a single study would have a significant impact on the results.

Country(ies) involved: China.

Keywords: anxiety; screening; mammography.

Contributions of each author:

Author 1 - Zhi-Gang Zhang - ZGZ conceived this study, designed the inclusion/exclusion criteria and the searching strategy, Will draft the protocol and revised the manuscript.

Author 2 - Li-Ping Yang - LPY conceived this study, Will draft the protocol and revised the manuscript.

Author 3 - Yi Shang - YS conceived this study, designed the inclusion/exclusion criteria and the searching strategy, will search for the literature, collect the data and made statistical analysis, and draft the protocol and revised the manuscript.

Author 4 - Zi-Wei Song - ZWS conceived this study, designed the inclusion/exclusion criteria and the searching strategy, will search for the literature, collect the data and made statistical analysis, and draft the protocol and revised the manuscript.

Author 5 - Li Du - LD will design a data extraction table. 